Original Article

\title{
ANATOMY IN ASYMPTOMATIC LUMBAR FACET JOINT ARTHROSIS
}

\author{
Ruchira Sethi*, Vishram Singh ${ }^{\star *}$ \\ *Department of Anatomy, Heritage Institute of Medical Sciences, Varanasi, UP, India \\ ${ }^{* *}$ Department of Anatomy, Santosh Medical College, Ghaziabad, UP, India
}

\begin{abstract}
Introduction: The facet joints are the only synovial joint of spine and undergo osteoarthritic changes referred to as facet joint arthrosis (FJA). The arthrosis is akin to arthritic changes in any other synovial joint of the body and may manifest as back pain or may remain asymptomatic throughout life. The causes for pain manifestation in FJA are manifold but there is paucity of literature in defining reasons to remain asymptomatic even with a deranged pathology at the joint. Thus the aim of the study was to assess the prevalence of asymptomatic FJA and define its relation with respect to age, gender and vertebral level on CT evaluation and discuss the pertinent anatomy around each spinal motion segment which prevents manifestation of pain.
\end{abstract}

Material \& Methods: A total of 100 asymptomatic subjects were selected on basis of a low back pain questionnaire within age range of 20-70 years, reporting to radiology department for CT abdomen. The individuals were categorized as per the following age groups; 20-35yrs as young; 36-50 as middle and 51-70 as old age groups. All five lumbar vertebral levels with their facet joints were analysed for evidences of degenerative grades and their severity, in total selected subjects. The images obtained were further assessed on image analysis software for distribution as per the age-groups, gender and prevalence with respect to vertebral level.

Results: A total of $136(27.2 \%)$ levels in $43(43 \%)$ spines were found to have FJA. The highest prevalence $(43.3 \%)$ of asymptomatic FJA was observed in the middle age group with involvement of multiple spinal motion segments and with grade 4 severity.

Conclusion: Prevalence of severe grades of asymptomatic FJA in middle age group suggests the possible role of certain muscle groups in maintaining an appropriate posture in spite of on-going degenerative processes.

Keywords: Facet joint, asymptomatic, lumbar, synovial, arthrosis, low back pain, para-spinal muscles.

\section{INTRODUCTION}

Facet joints play a significant role in spinal biomechanics by contributing largely to rotational kinematics of the spine. The task is greater in terms of lumbar facet joints where the robust lumbar vertebrae bear the maximum weight of the body and also limit the spinal rotation due to fixed pelvis. The role of lumbar facet joints in rotational kinematics is variable, depending on the normal and deranged anatomy of the three components respective to a spinal motion segment. Each spinal motion segment includes the anteriorly placed intervertebral disc (IVD) and the posteriorly located two synovial facet joints (FJs), together referred to as the "tripod joint" [1,2]. Each spinal motion takes both compressive and rotational component of the load during postural alignment. The compressive load is borne by the anterior fibres of annulus fibrosus of the disc while the rotational component is distributed equally to the two FJs $[1,3]$. The mutual interplay of the disc and facet joint makes it debatable to define the prime event in degenerative cascade of the spine.

Many are of the view that disc is the prime component to undergo degeneration and manifests as radiating, debilitating low back pain in individuals [4].

\section{Address for Correspondence:}

Dr Ruchira Sethi, Associate Professor, Department of Anatomy, Heritage Institute of Medical Sciences, Bhadwar, NH2 bypass, Varanasi, UP, India. Mob: 9873621520 Email: ruchirasethi@gmail.com 
In a normal motion segment, the outermost fibres of annulus fibrosus (AF) are the first component to bear the load and rotational strain. These fibres are made up of concentric layers of collagen type I fibres and hold the gelatinous nucleus pulposus (NP) within, which is predominantly collagen type II fibres [5]. This arrangement of fibres peripherally and centrally provides the discs the capacity to bear the maximum strain during static and normal kinematic alignment of the spine. But during excessive weight/load bearing and abrupt kinematic changes there occurs tears within the AF, progressing to fraying and dehydration of the NP with eventual loss of the annular-nuclear distinction [6,7]. Horst and Brinckmann (1980) demonstrated that severely degenerated discs load asymmetrically under eccentric compressive conditions, reflecting the solid-like behaviour of degenerated discs in contrast to the fluid-like properties of healthy discs [8]. This solid -like disc distributes the load asymmetrically which eventually causes disrupted load distribution to the posterior component of the spine, the facet joints. Yang and King (1984) predicted a significant rise in facet joint loading pressure with increasing segmental degeneration using a finite element model of disc degeneration (DD) [9]. This thus suggests that the discs are the first to be involved in the degeneration of spine followed by degeneration of facet joints and gradually manifests as low back pain (LBP). However there have been cases with evidences of DD in otherwise asymptomatic individuals also and this has been debated with reference to avascular and aneural nature of the disc $[10,11]$. This probably suggests that DD in itself is not the only source of pain but some other factor/ factors contribute towards patient symptomatology.

Secondly, the facetogenic theory suggests the facets joints to be the prime pain generator in the spinal degenerative cascade. These joints are typical diarthrodial joints which may be $\mathrm{C}$ or $\mathrm{J}$ shaped having structure similar to any other large synovial joint of the body. The fibrous capsule, articular cartilage, joint cavity with potential joint space of 2-4mm, filled with 1$2 \mathrm{ml}$ of joint fluid is the normal anatomy of the joint (Fig. 1) [12].

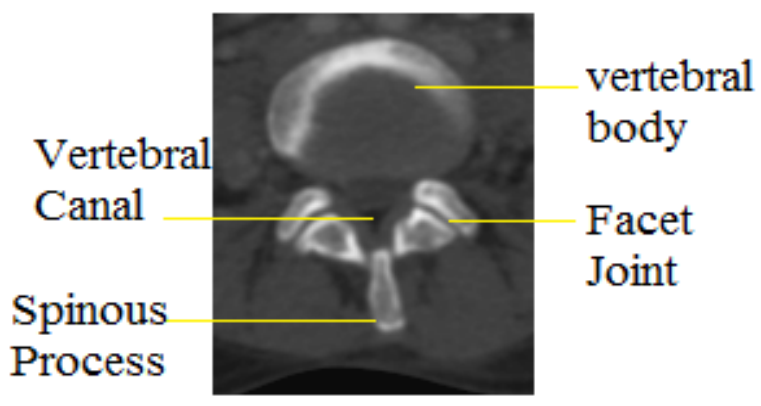

Fig.1: Normal facet joint on CT imaging

Owing to their postero-lateral location and structural anatomy these joints depend largely on the spine's response and are also able to alter the spines' response during conditions of mechanical load bearing. Due to normal process of ageing the joints undergo osteo-arthritic changes similar to any other synovial joint in the body [13]. The location of low threshold mechanoreceptors, nociceptor fibres in the fibrous capsule and surrounding structures it contributes largely to pain manifestation during the process of arthrosis. Also the release of inflammatory mediators in the joint cartilage has been appreciated during degenerative joint anatomy and all these reasons are sufficient enough to produce facetogenic pain [14]. Various epidemiological studies for pain associated with FJA have however provided conflicting results. An extensive work of Eubunks et al. (2007) has defined facet arthrosis as a universal finding in individuals as young as less than 20 years [15]. There have been studies where severe grade of arthrosis is evident in otherwise asymptomatic subjects [16, 17]. This once again suggests that the facet joint alone is insufficient to produce symptoms and clinical manifestations of LBP. Hence the term FJA is more of pathology at the FJ with cartilage destruction, osteophyte formation, decrease in joint space rather than a clinical entity, as the manifestation of pain occurs only after involvement of second component (IVD) of the spinal motion segment.

Throughout the whole spine, the lumbar region is better aligned to bear the mechanical load because of the larger size of the vertebral body, curvilinear shape of articular surface and more sagittal orientation of the lumbar facet joints [18]. The flexion-extension and rotational movements are restrained at the lumbar spine due to more sagittal orientation of the facet joints as compared to cervical and thoracic segments. This 
in turn, acts as a protective mechanism to prevent extensive shear and torsional force component on the IVDs. Thus, in the lumbar region, it would be inappropriate to suggest that the disc degeneration is the prime event in the degenerative cascade. The present study is thus an attempt to detect the prevalence of lumbar facet arthrosis in otherwise healthy asymptomatic subjects with no other radiological or imaging evidences of disc degeneration at the lumbar vertebral levels.

Various studies on pathophysiology of LBP have documented either the disc or the facets as the source of pain but very few studies till date have discussed the reasons for subjects to remain asymptomatic even with on-going degenerative process at either of the spinal motion component. There might be some other anatomical factors that may contribute to maintain the spinal posture, stability and integrity of spinal biomechanics even with deranged anatomy of the facet joints. Keeping in view the consideration of evidences of asymptomatic FJA in lumbar region, the aim of the study was to define the prevalence of FJA in healthy, asymptomatic (for LBP) Indian population, its relation to age, gender and level of vertebral involvement and suggest probable causes or factors responsible to maintain an asymptomatic condition even with a deranged joint.

\section{MATERIALS AND METHODS}

The study was executed after necessary institutional ethical clearance letter was obtained. It was conducted at Santosh Medical College, Ghaziabad in collaboration with Safdurjung Hospital, New Delhi. The study was conducted on subjects reporting to radiology department for $\mathrm{CT}$ abdomen for diseases other than that of spine. Only subjects who gave a written informed consent were chosen for the study. The individuals were categorized as per the following age groups; $20-35$ yrs as young; $36-50$ as middle and 51-70 as old age groups. A modified Nordic low back pain questionnaire was provided to the subjects to categorize as asymptomatic and symptomatic for LBP [19]. It was a questionnaire designed to have a binary or multiple choice response. The first question in the series was designed to categorize the patient as symptomatic or asymptomatic followed by series of questions to eliminate the possibility of false positive response. Once categorized as per symptomatology, asymptomatic subjects were taken up for CT scan. The CT scan was performed on subjects in a standard protocol in supine position on Philips brilliance 40
(120kV 250mAsCT $8{ }^{\star} 2.5$ slice collimation). The scans obtained from 100 asymptomatic subjects were taken for further interpretation on image analysis software (Philips DICOM viewer R3.0). Only those images with no evidences of disc degeneration were taken up for the study.

Any spine having FJA evidences at one or more than one vertebral level was taken as arthritic spine. Subsequently, selected subjects with no symptoms for LBP and positive evidences of degeneration at FJs were taken for further analysis. The facet joint morphology was re-assessed from L1-L5 motion segments to grade the severity of arthritic changes. The classification of Pathria et al. (1987) was used to categorize the disease severity from least severe as grade 1 (with joint space less than $2 \mathrm{~mm}$ ) and most severe as grade 4 (with complete ankylosis) [20] (Fig. 2). It has been documented earlier that the arthrosis is independent of side (right or left), thus any one side involvement and side with higher severity grade was taken as the grade for FJA [21]. The results for FJA presence and disease grades were computed with respect to gender, age groups and vertebral level. SPSS 19 was used for statistical computation. The confidence interval $(\mathrm{Cl})$ was set at $95 \%$ and $p$ value less than 0.05 was taken as significant association.
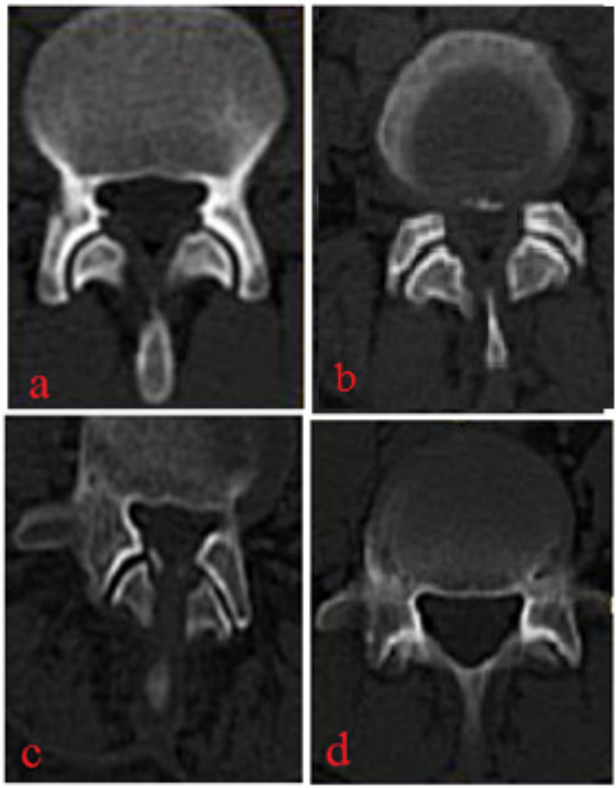

Fig. 2: Degenerative grades of FJA

$a=$ grade 1 (decreased joint space), $b=$ grade 2 (hypersclerosis of joint margins), $c=$ grade 3 (presence of osteophytes, $d=$ grade 4 (complete ankylosis) 


\section{OBSERVATIONS AND RESULTS}

From the questionnaire interpretation, 100 subjects with 44 males and 56 females were selected for the study. There was no statistically significant difference of age between genders ( $p>0.05, \mathrm{Cl} 95 \%)$. Out of 100 , CT images of 43 (21 males \& 22 females) spines; mean age $44.26 \pm 12.85$ years; had osteo-arthritic changes at the FJs with no evidences of disc degeneration. These spines were categorized as arthritic spines and used for further analysis. The mean age for males and females was $46.24 \pm 12.4$ years and $42.6 \pm 13.3$ years respectively, with no statistically significant difference of age between the genders; $p>0.05$ at $95 \% \mathrm{Cl}$.

The analysis at each spinal motion level for FJA was done for the selected 43 spines (215 spinal motion levels) and a high prevalence (63.2\%) for FJA was observed in asymptomatic subjects. The results at each vertebral level suggest highest involvement of L4-L5 followed by L5-S1 level. The prevalence gradually decreased rostrally from L4-L5 and the results were statistically non-significant with respect to vertebral level (Table 1).

Table 1: FJA prevalence at different vertebral levels for asymptomatic subjects

\begin{tabular}{|l|c|c|c|}
\hline Vertebral Level & FJA +ve (\%) & FJA -ve (\%) & P value \\
\hline L5-S1 & $34(15.8)$ & $9(4.1)$ & \\
\cline { 1 - 2 } L4-L5 & $35(16.3)$ & $8(3.8)$ & \multirow{2}{*}{0.39} \\
\cline { 1 - 2 } L3-L4 & $26(12.1)$ & $17(7.9)$ & NS \\
\cline { 1 - 2 } L2-L3 & $22(10.2)$ & $21(9.8)$ & \\
\cline { 1 - 2 } L1-L2 & $19(8.8)$ & $24(11.2)$ & \\
\hline TOTAL & $\mathbf{1 3 6 ( 6 3 . 2 )}$ & $\mathbf{7 9 ( 3 6 . 8 )}$ & \\
\hline
\end{tabular}

The presence of arthrosis was further analysed with respect to genders (Fig. 3). Females had a higher proportion of FJA at each vertebral level but the results were statistically non-significant between genders $(p=0.964, \mathrm{Cl} 95 \%)$.

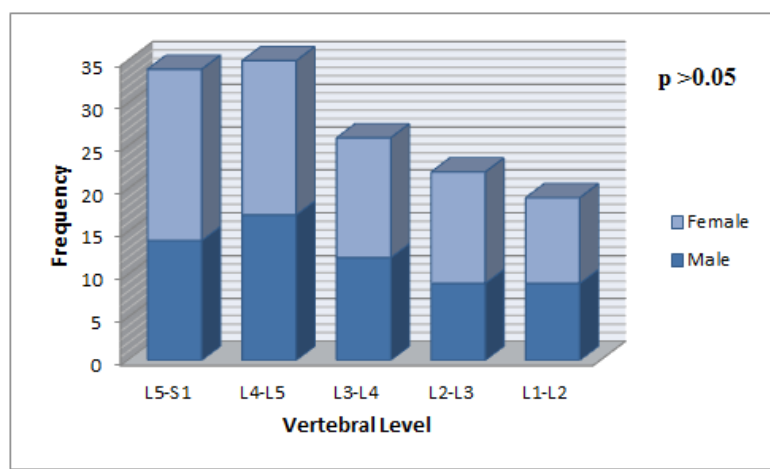

Fig. 3: Proportion of FJA cases between genders for asymptomatic subjects

The distribution of FJA positive cases as per the defined age groups was found to be statistically independent of age groups when considered at each vertebral level. Hence all the vertebral levels were studied in total with respect to age groups and it was observed that the highest prevalence was in the middle age group and was statistically highly significant $(p<0.01, p=0.001)$ (Fig. 4). This suggests that degeneration of facet joints is dependent on the age of an individual, highest being at middle age groups, but still remaining asymptomatic.

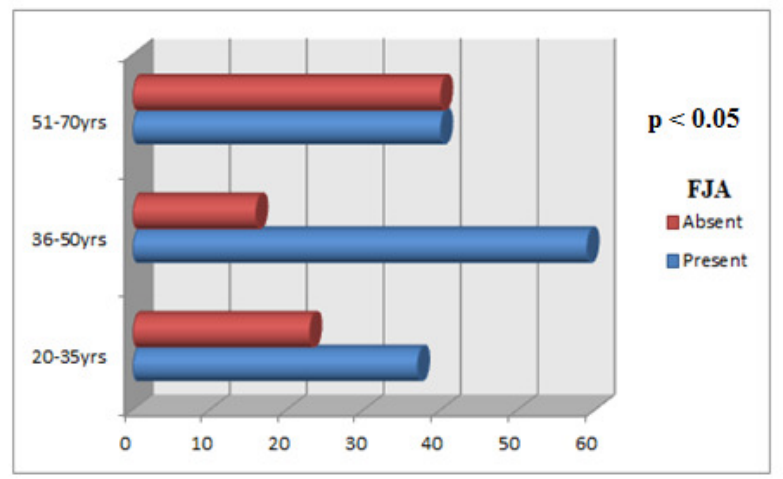

Fig. 4: Distribution of FJA cases in different age groups

Another observation was that even with multisegmental involvement in one spine with most severe degenerative grades the patient remained asymptomatic (Fig. 5). The highest proportion was for grade 2 FJA at each vertebral level. 


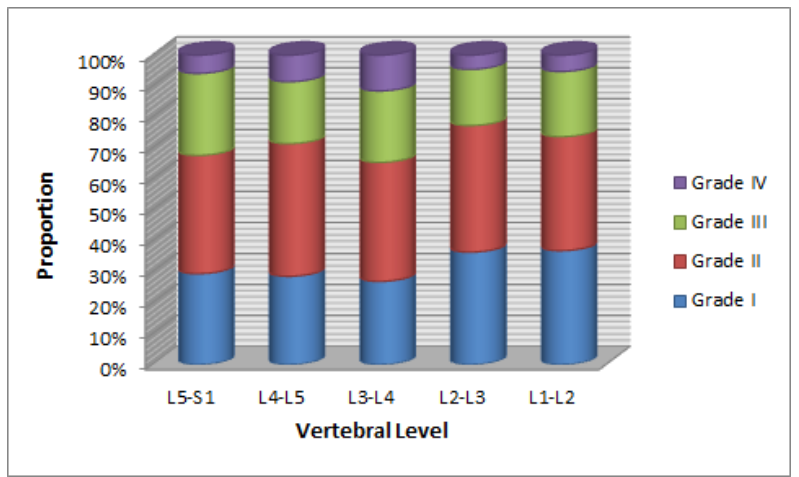

Fig. 5: Severity grades for FJA at different vertebral levels

\section{DISCUSSION}

A high prevalence $63.2 \%$, of facet joint arthrosis, was seen in asymptomatic subjects and almost similar results have been reported by earlier researchers [1, 22]. The L4-L5 spinal motion level was most frequently affected in the present study and Eubanks et al. (2007) on 647 cadaveric lumbar spines also concluded with similar findings [15]. A high proportion of arthrosis at L4-L5 as compared to L5-S1 is explained by the fact that the large L5 vertebral body supported by strong osseo-ligamentous structures allow minimal rotational movements to occur at this level, preventing arthrosis. The fixed pelvis (sacral body) on which L5 rests spares L5-S1 segment from abrupt postural changes and subsequent degeneration [22]. Facet tropism, which may be a probable cause of degeneration, is defined as the angular asymmetry between the left and right facet joint orientation and is also commonly reported at L4-L5 level [23, 24].

The higher prevalence observed in females in the present study is explained by the fact that women have a higher body mass index which otherwise increases lumbar lordosis. The increase in lumbosacral angulations due to exaggerated lordosis consequently increases the load on the facet joints leading to their degeneration [25]. Also, higher prevalence in females is attributed to estrogen which has high chondro-destruction ability [26, 27]. Histological studies of facet articular cartilage have observed increase number of estrogen receptors in cases with FJA. The mechanical load bearing by the articular cartilage is equally distributed throughout the surface of the cartilage in a static spine but during kinematic flexion-extension movements increased load is borne by the superior and inferior poles of the cartilage. This is proved by various studies which have documented sparing of the central part of the cartilage, with majority degenerative changes occurring at the margins of the articular cartilage $[28,29]$.

A significant association between age groups and asymptomatic FJA was observed in the present study. Osteo-arthritis, in any synovial joint, is an age-related degenerative process, it follows logically that advanced arthritis might be associated with pain in older adults, and not in younger adults [30, 31]. A higher prevalence of asymptomatic FJA in middle age group suggests the possible role of para-spinal muscles in maintaining the structural integrity and functional stability even in presence of arthrosis. With ageing there occurs a reduction in muscle cell number, muscle twitch time and twitch force, decrease in sarcoplasmic reticulum volume and calcium pumping capacity thus patients in older age groups start presenting the pathology as a disease entity with manifestations of painful symptoms [32, 33]. The level of physical activity which usually decreases with age has also been stated as an important factor for decreased muscle mass. The role of physical activity is manifested by a study on tennis players where early and severe degeneration was observed without any clinical complaints [16]. The spinal musculatures not only provide structural support and prevent buckling of vertebral column but also provide proprioceptive feedback regarding the position of spine in space [17, 34]. With increasing age, there is decrease in paraspinal muscle mass which contributes significantly to FJA and also impaired spinal proprioception evident as chronic low back pain [34, 35].

The osteophyte formation in a facet joint is seen even in grade 2 type of disease. The mere presence of osteophytes is not suggestive of disease severity and neither is indicative of clinical symptoms. The formation of osteophytes is considered as a remodelling phenomenon and may or may not be seen even in severe cases of degeneration, thus their mere presence does not relate to disease severity [17, 36]. The location of osteophytes with or without disc involvement suggests some cases of asymptomatic arthrosis even with grade 4 variant. Intervertebral foramen is a tear drop shaped exit for the neurovascular structures. Unless there is decrease in height or width of IVF, there are hardly any chances of compression of structures passing through it. The height of the foramen is largely dependent on the discal height which was not affected in our study groups. The formation of osteophytes at the superior margin of the articular facet also spares the nerves which occupy the posterior upper part of the foramen. 


\section{CONCLUSION}

The FJA is a pathological event which is evident even in younger age groups. It is independent of vertebral level and gender but dependent on age. The increased muscle mass and physical activity in middle age groups probably are the reasons for the subjects to remain asymptomatic even with most severe form of degenerative pathology at multiple spinal segments. The increased surface area of the articular surface of caudal lumbar vertebrae and specific site for osteophytes are also factors which spare the subject from having LBP.

\section{REFERENCES}

1. Kalichman L, Hunter DJ. Lumbar facet joint osteoarthritis: A Review. Semin Arthritis Rheum. 2007; 37(2): 69-80.

2. Singh V, Sethi R. Lumbago and associated morbid anatomy of lumbar spinal canal and facet joints. J Anat Soc India. 2014; 63(1): 77-84

3. Inoue N, Espinoza Orías AA. Biomechanics of intervertebral disk degeneration. Orthop. Clin. North Am. 2011; 42:487-499.

4. Deyo RA, Weinstein JN Low back pain. N Engl J Med. 2001; 344(5):363-70.

5. Roberts S, Menage J, Duance V, Wotton S, Ayad S.1991 Volvo Award in basic sciences. Collagen types around the cells of the intervertebral disc and cartilage end plate: an immunolocalization study. Spine (Phila Pa 1976). 1991; 16(9):1030-8.

6. Pollintine P, Przybyla AS, Dolan P, Adams MA. Neural arch load-bearing in old and degenerated spines. J Biomech. 2004; 37:197-204.

7. Sato K, Kikuchi S, Yonezawa T. In vivo intradiscal pressure measurement in healthy individuals and in patients with on going back problems. Spine. 1999; 24: 2468-74.

8. Horst M, Brinckmann P. 1980 Volvo award in biomechanics: measurement of the distribution of axial stress on the end-plate of the vertebral body. Spine (Phila Pa 1976). 1981; 6:217-232.

9. Yang $\mathrm{KH}$, King Al. Mechanism of facet load transmission as a hypothesis for low-back pain. Spine. 1984; 9(6):557-65.

10. Kim SJ, Lee TH, Lim SM. Prevalence of disc degeneration in asymptomatic Korean subjects. Part 1: Lumbar Spine.J Korean Neurosurg Soc. 2013; 53(1), 31-38.

11. Binch ALA, Cross AK, Le Maitre CL. The regulation of nerve and blood vessel ingrowth in aneural and avascular intervertebral disc and articular cartilage. OA Arthritis. 2014; $2(1): 4$.

12. Ko S, Vaccaro AR, Lee S, Lee J, Chang $H$. The prevalence of lumbar spine facet joint osteoarthritis and its association with low back pain in selected Korean populations. Clin Orthop Surg. 2014; 6(4), 385-391.
13. Weishaupt D, Zanetti W, Boos N, Hodler J. MR imaging and CT in osteoarthritis of the lumbar facet joints. Skelet Radiol. 1999; 28:215-219.

14. Cavanaugh JM. Pain generation in lumbar and cervical facet joints J Bone Jointt Surg Am. 2006; 88 (Suppl 2): 63-67.

15. Eubanks JD, Lee MJ, Cassinelli E, Ahn NU Prevalence of lumbar facet arthrosis and its relationship to age, sex, and race: an anatomic study of cadaveric specimens. Spine (Phila Pa 1976). 2007; 32(19):2058-2062.

16. Alyas F, Turner M, Connell D. MRI findings in the lumbar spines of asymptomatic, adolescent, elite tennis players. $\mathrm{Br} \mathrm{J}$ Sports Med. 2007; 41(11), 836-841.

17. Gellhorn AC, Katz JN, Suri P. Osteoarthritis of the spine: the facet joints. Nat Rev Rheumatol. 2013; 9(4), 216-224.

18. Panjabi MM, Oxland T, Takata K, Goel V, Duranceau J, Krag M. Articular facets of the human spine. Quantitative three dimensional anatomy. Spine (Phila Pa 1976). 1993; 18(10): 1298-1310.

19. Kuorinka I, Jonsson B, Kilborn A, Vinterberg $H$, BieringSorensen F, Anderson G, Jorgensen K. Standardised Nordic questionnaires for the analysis of musculoskeletal symptoms. Appl Ergon 18(3):233-7.

20. Pathria M, Sartoris DJ, Resnick D. Osteoarthritis of the facet joints: accuracy of oblique radiographic assessment. Radiology. 1987; 164(1):227-30.

21. Rong X, Liu Z, Wang B, Pan X, Liu H. Relationship between facet tropism and facet joint degeneration in the sub-axial cervical spine. BMC Musculoskelet Disord. 2017; 18(1):86.

22. Taheri A, Moallemy A, Dehghanian A, Vatankhah P. Evaluation of the relationship between age, gender, and body mass index, and lumbar facet joint pain. Indian J Pain. 2016; 30:19-22.

23. Alonso Fernando, Hristina M, William J, Christian F, Sameera U, Tarush Rustagi, Marios Loukas, Jens R. Chapman, Rod JO, R. Shane Tubbs. Lumbar Facet Tropism: A Comprehensive Review. World Neurosurgery 2017; 102:91-96.

24. Shin MH, Ryu KS, Hur JW, Kim JS, Park CK. Association of facet tropism and progressive facet arthrosis after lumbar total disc replacement using ProDisc-L. Eur Spine J. 2013; 22(8):1717-22.

25. Been E, Kalichman L. Lumbar Lordosis. Spine J. 2014; 14(1):87-97.

26. Felson DT, Nevitt MC. The effects of estrogen on osteoarthritis. Curr Opin Rheumatol. 1998; 10:269-272.

27. Ha KY, Chang $\mathrm{CH}$, Kim YS, Kim KW, Na KH, Lee JS. Expression of estrogen receptor of the facet joints in degenerative spondylolisthesis. Spine. 2005; 30(5):562-566.

28. Moore RJR, Crotti TNT, Vernon-Roberts B. Osteoarthrosis of the facet joints resulting from anular rim lesions in sheep lumbar discs. Spine (Phila Pa 1976). 1999; 24: 519-525.

29. Li J, Muehleman C, Abe Y, Masuda K. Prevalence of facet joint degeneration in association with intervertebral joint degeneration in a sample of organ donors. J. Orthop. Res. 2011; 29:1267-1274.

30. Lethbridge-Cejku M, Scott WW Jr, Reichle R, Ettinger WH, Zonderman A, Costa P, Plato CC, Tobin JD, Hochberg MC. 
Association of radiographic features of osteoarthritis of the knee with knee pain: data from the Baltimore Longitudinal Study of Aging. Arthritis Care Res. 1995; 8:182-188.

31. Borenstein D. Does osteoarthritis of the lumbar spine cause chronic low back pain? Curr Pain Headache Rep. 2004; 8:512517.

32. Vandervoort AA. Aging of the human neuromuscular system. Muscle Nerve. 2002; 25:17-25.

33. Lexell J. Human aging, muscle mass, and fiber type composition. J Gerontol A Biol Sci Med Sci. 1995; 50: 11-16.

34. Leinonen $V$, Kankaanpa $M$, Luukkonen $M$, Kansanen $M$, Hanninen $\mathrm{O}$, Airaksinen $\mathrm{O}$, Taimela S. Lumbar paraspinal muscle function, perception of lumbar position, and postural control in disc herniation-related back pain. Spine (Phila $\mathrm{Pa}$ 1976). 2003; 28:842-848.

35. Kalichman L, Hodges P, Li L, Guermazi A, Hunter DJ. Changes in paraspinal muscles and their association with low back pain and spinal degeneration: CT study. Eur. Spine J. 2010; 19:1136-1144.

36. Eisenstein SM, Parry CR. The lumbar facet arthrosis syndrome. Clinical presentation and articular surface changes. J Bone Joint Surg Br. 1987; 69:3-7. 